\title{
Mobile ACORoute: Route Recommendation Based on Communication by Pheromones
}

\author{
Carla S. G. Pires, Marilton S. de Aguiar, and Paulo R. Ferreira \\ Centro de Desenvolvimento Tecnológico, Universidade Federal de Pelotas (UFPEL), \\ Brazil
}

\begin{abstract}
Urban mobility problems affects the vast majority of cities nowadays. Thus, systems that provide real time information to assist in planning routes and choosing the most appropriate paths are essential to make transport more effective. As an alternative solution to problems related to mobility in cities, there are the so-called Intelligent Transportation Systems (ITS) which include the Route Recommendation Systems (RRS) and methodologies for congestion prediction that combine Information and Communication Technology (ICT) with Artificial Intelligence (AI) technonology to improve the quality of transport systems. In this context, this work proposes the use of pheromone-based communication for building an ITS that offers information about real time traffic flow, taking into account the mobility of vehicles and passengers and the traffic dynamics. The general goal is to provide an Android solution able to suggest users routes calculated by the hybrid algorithm between $\mathrm{A}^{*}$ and pheromone mechanism. The idea is to avoid areas of heavy traffic congestion.
\end{abstract}

Keywords: Route recommendation systems, intelligent transportation systems, pheromone based communication.

\section{Introduction}

In the past decades, the traffic in medium and large cities, as well as the incovenience caused directly or indirectly by it, cause increasing mobility problems. The Intelligent Transportation Systems (ITS) are shown as an alternative to improve mobility within cities through the application of Information and Communication Technology (ICT) to support the existing traffic infrastructure and improve the quality of transport systems [17].

A wide variety of ITS tools have played important roles in the effectiveness of transport. These systems provide information related to traffic, influencing in various aspects of transport in relation to urban mobility. Most of these ITS tools uses static information aided by the traffic infrastructure integrated technologies [5]. This article proposes to use information from mobile devices to dynamically determine the best path for the driver seeking to avoid traffic congestion.

To this end, this paper proposes an approach for the calculation of the trajectory based on the use of pheromone dynamics. Other approaches to congestion prediction using Swarm Intelligence have been propose $[10,11,12]$. Previous works differ in how pheromones influence the calculation process and do not consider some aspects of their practical application.

The paper is arranged as follows: in Section 2 the theoretical background are briefly introduced; Section 3 discusses the conceptual and technological foundations of the work; Section 4 discusses about the development of ACORoute and shows how the proposed approach was implemented; 5 presents the results obtained from the application of the proposed approach in

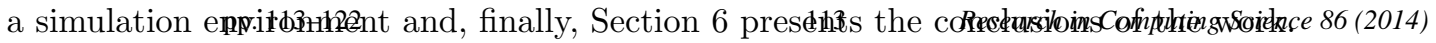




\section{Rerlated G Porkilton S. de Aguiar, and Paulo R. Ferreira}

\subsection{Intelligent Transportation Systems (ITS)}

The term ITS emerged in USA in the late 1980s with a movement aiming to make transport safer, more effective and reliable. The ideia of the ITS is to support the existing traffic infrastructure without the need to change it [1]. Thus, a number of tools have been developed in order to assist drivers to stay informed about the traffic conditions and the relevant aspects of the dynamics of its flow. Areas of Computing as Artificial Intelligence (AI), Computer Vision, Pattern Recognition, Machine Learning, Data Mining, and Intelligent Control have been intensively explored in this process [14].

The tools developed in ITS enable users to get several kinds of information related to traffic. This information range from current location, alternative routes, road conditions, even weather forecasts along the route, providing greater power of decision on the actions and choices of individuals.

In a simplified way one can say that the ITS aim at the optimization of existing transport systems by making use of a wide range of tools that combine technologies and improvements in information systems, communications, sensors and advanced mathematical methods. The objective is to obtain roads, vehicles and more "intelligent" users, with an attempt to facilitate the flow of traffic, and solve a variety of transportation problems of our days, such as congestion, safety and environmental problems [16].

\subsection{Swarm Intelligence}

An interesting approach is the use of techniques of swarm intelligence, as the organization of bee colonies and allocation of tasks among insect societies. The dynamics of insect society are the result of different actions between the population and the environment. This interaction between agents creates a system of communication that contributes to the formation of "Collective Intelligence" assigned to the insect society. This technique arose from observations and studies of the behavior of living beings usually searching for food [4].

Swarm intelligence or collective intelligence has been successfully applied in dynamic optimization problems in various fields, such as the traveling salesman problem, quadratic assignment problems, load balancing and vehicle routing problems. These are just some examples where swarm intelligence is applied [15].

Examples of applications of this nature can be seen in [2], which introduced systems of bees as a new approach in the area of intelligent swarms applied to problems of transportation engineering. They developed a new heuristic for the traveling salesman problem by defining an artificial environment of bees; swarm intelligence technique inspired by the behavior of ant colonies are also used to optimize the timing of traffic lights [6]; and, the use of the swarm intelligence inspired by the behavior of bees society for task allocation using clustering, grouping agents by skills, considering societies of bees and how they collect the best nectar from the available sources through simple rules of behavior [13].

The use of intelligent insect swarms has already been applied in problems to find shorter routes between cities (TSP), such as [9], who developed a new heuristic for the traveling salesman problem by setting an artificial environment of bees, where each bee is an agent performing activities defined by the model and the communication occurs in the interaction between them [8].

This work has focused on ant colony optimization (ACO) that is inspired by the observation

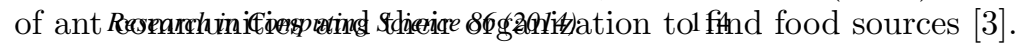


Experiments were conducted to ACORoute- Route Recommendation Based on Communication by Pheromones ability to discover the shortest path very quickly. It was observed that only the first ants randomly choose the path, thus those who choose the shorter path arrive faster to the nest. Thus, the probability of choosing the shortest way increases continuously and rapidly all ants start using the shortest path [15].

The ACO is based on this behavior, considering that each ant walking on a trail deposits certain amount of pheromone in it, then the next ants follow the path with a proportional probability to the amount of pheromone present in this path and thus reinforcing the current pheromone [3].

ACO was the first swarm intelligence algorithm to be developed, it is used in the development of this work. It has been effectively applied to solve the traveling salesman problem, as well as several other problems related to Transportation and Traffic Engineering. [7] and [11] used the swarm intelligence technique inspired in the behavior of ants society (ACO). They employed this methodology for predicting traffic congestion in a simulated environment, considering agents (sensors) installed at the intersections of routes that manage information of pheromones. [2] evaluated the application of pheromone communication in real traffic conditions applied to congestion prediction and observed that the method is effective for short-term predictions.

\section{Mechanism for Congestion Identification Based on Communication by Pheromones}

Technologies for congestion identification are a key element to support Intelligent Transportation Systems. Currently, many methods have been proposed, many of them using collective intelligence, based on the idea that societies of insects perform complex tasks using decentralized communication based on pheromones. In this context, pheromones are considered as a means to provide information $[2,7,11,12,18]$ among others.

in this paper we are proposing congestion identification using the technique of communication based on pheromones (ACORoute), which was also proposed by [2], [7] and [11] in their works. Different from the adopted in our work, they use the infrastructure provided by local transport system and/or devices installed in vehicles to collect, process and store data related to traffic. In addition, when calculating the route, ACORoute uses the pheromone information in heuristic of the best route, while the papers presented use a common navigator to calculate, merely use pheromones for congestion prediction. A comparison between relevant works can be seen in Table 1.

when dealing to congestion prediction, the application WAZE should be mentioned, which recommends routes avoiding places of heavy flow. However, it only offers the collaboration of other users who interact with the application to report occurrences. Thus, apart from Waze (2013), which also makes congestion prediction, other works have results only in simulated environment to date to validate their technical environments. There are no real applications.

\section{ACORoute}

\subsection{Pheromone-Based Model}

The use of pheromone dynamics for congestion identification was discussed in the development of ACORoute, and it was proposed a model that predicts congestion based in the pheromones com-

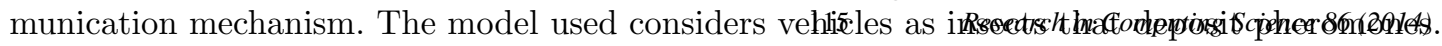


Carla S. G. Pires, Marilton S. de Aguiar, and Paulo R. Ferreira

Table 1: Characteristics of the Method for Congestion Prediction. Where Ph stands for pheromone.

\begin{tabular}{|c|c|c|c|c|c|c|}
\hline & $\begin{array}{c}\text { Self-organising } \\
\text { Narzt }(2010)\end{array}$ & $\begin{array}{l}\text { Pheromone } \\
\text { Ando (2006) }\end{array}$ & $\begin{array}{c}\text { Traffic-Congestion } \\
\text { Kurihara (2013) }\end{array}$ & $\begin{array}{l}\text { Hybrid ACO } \\
\text { Ochiai (2014) }\end{array}$ & $\begin{array}{c}\text { WAZE } \\
\text { WAZE (2013) }\end{array}$ & $\begin{array}{c}\text { ACORoute } \\
\text { Us }\end{array}$ \\
\hline Algorithm & - & $\mathrm{Ph}$ & - & Dijkstra & $\mathrm{A}^{*}$ & $\mathrm{~A}^{*} / \mathrm{Ph}$ \\
\hline Context data & No & No & No & No & No & Yes \\
\hline Mobile & No & No & No & No & Yes & Yes \\
\hline $\begin{array}{r}\text { Sources of } \\
\text { information }\end{array}$ & No & Yes & Yes & Yes & Yes & Yes \\
\hline $\begin{array}{r}\text { Traffic } \\
\text { infrastructure }\end{array}$ & Yes & Yes & Yes & No & No & No \\
\hline $\begin{array}{r}\text { User } \\
\text { preferences }\end{array}$ & No & No & No & Yes & No & Yes \\
\hline $\begin{array}{r}\text { Historical } \\
\text { data }\end{array}$ & No & No & No & No & Yes & Yes \\
\hline Real Time & No & No & Yes & No & No & Yes \\
\hline $\begin{array}{r}\text { route } \\
\text { re-planning }\end{array}$ & Yes & No & No & No & No & Yes \\
\hline
\end{tabular}

The operating principle of this strategy occurs with vehicles that mark their path by dropping digital pheromones which are perceived by all the vehicles that travel in the environment. This mechanism is used to allow the calculation of a route that avoids heavy traffic.

The vehicle sends information about geopositioning to the server at every 30 seconds and with this information is created of the pheromone map, that is built as follows: when the georeferencing information is sent by the application it is received by the WebService, the value of 0.8 is incremented at the referenced node (Latitude and Longitude).

When a given node remains without receiving information from pheromones longer than 30 seconds, the evaporation process starts until the amount of pheromones in the node reaches 0 . The value of the decrement to pheromone is 0.3 . These values were calibrated through simulation, which confirmed to be the most effective.

\subsection{The Best Route Algorithm}

To determine a route between a source node (latitude and longitude) and a destination node (latitude and longitude), the solution presented here uses a variation of the routing heuristic in graphs $\mathrm{A}^{*}$, considering besides the distance, the amount of pheromones in this route. The proposed algorithim prefers routes with lower level of pheromones

The map stored in the database is represented by a directed graph with weights representing distances. We can say that the graph is a logic representation of the map, containing the streets of the city of Pelotas, each section of the street is considered an edge and the points are nodes. The search algorithm $\mathrm{A}^{*}$, that considers the pheromone information to calculate the route, is used to find the best route between two points. The database used is derived from the open database of the Open Street Maps only considering the points inside the area of Pelotas.

\subsection{Android Application}

For the development of the application, the Eclipse IDE (Integrated Development Environment) combined with Android SDK (Software Develpoment Kit), that provides an API (Application

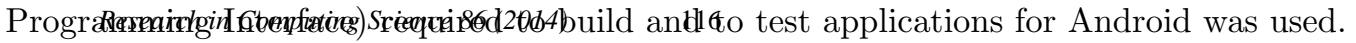




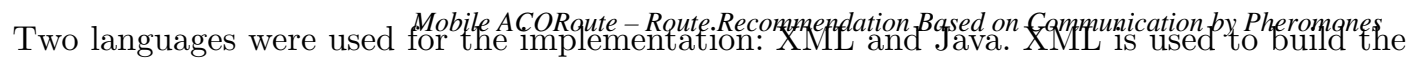
graphical part of the application, where the interaction with the user happens, made through the components of the graphic application, the widgets, such as buttons and textboxes. In the control, the Android-Java language, a standard Java subset, which implements specific API for Android application development was used.

In the Android API Activity and Service concepts that are related to the graphical interface of the application are defined which are responsible for the different screens, operations performed in the background, among them communication with the server.

The implementation project of the application was divided into two stages: i) receipt and transmission of georeferenced data, used to establish the historical base and the construction of the pheromone map, these information are obtained through GPS (Global Positioning System) from the device itself and ii) development of the interface with the user where information such as location and path of the calculated routes will be presented.

A service responsible for connecting the application to the GPS, receiving location information periodically every 30 seconds, which can be set remotely from the WebService was created in the first stage of the work. After receiving the information, Service sends it to the WebService, keeping the historical database and pheromones. The application prototype interface is shown in Figures $1 \mathrm{a}, 1 \mathrm{~b}$ and $1 \mathrm{c}$.

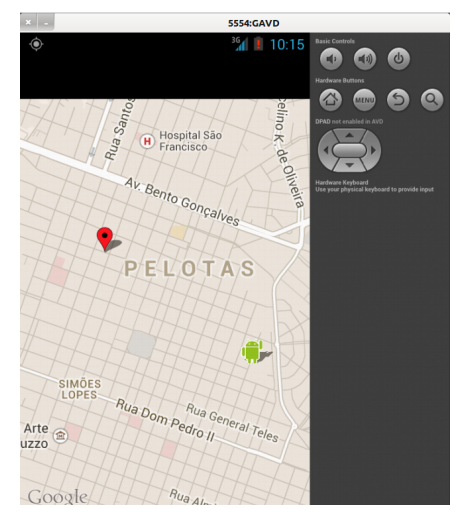

(a) Origin and destination points

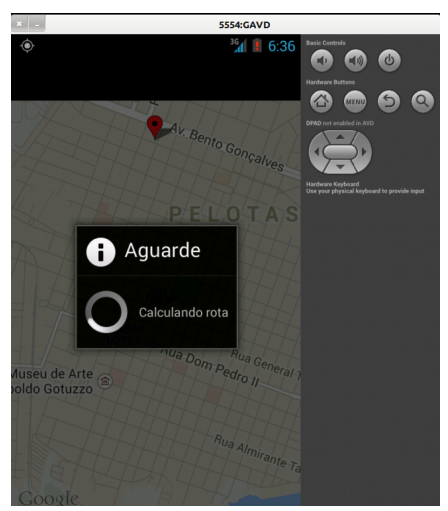

(b) Calculating the route

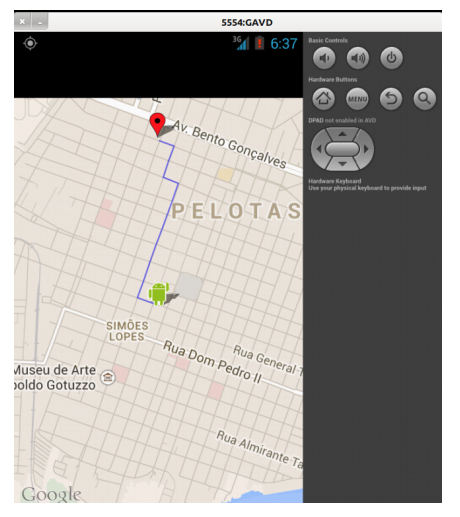

(c) Route view

Fig. 1: Prototype Interface.

The time to update the users location was defined in order to optimize the battery consumption of the device without sacrificing the accuracy of the application. When using smaller and more frequent values, it creates several accesses to the GPS which is not considered a good strategy for the substancial increase of battery consumption of the device. In contrast, using higher values can lead to a possible loss of information because it becomes complex to deduce the route taken by the user.

In the second stage of development, two activities were created, the first one, responsible for controlling the transmission of location information and for opening the map; and the second, responsible for displaying the map and all the relevant information, such as location of the user, menus and routes. The map chosen for the application was Google Maps, because it is easy to use and complete, in addition to having its own API for Android programming, which makes the implementation simpler. 


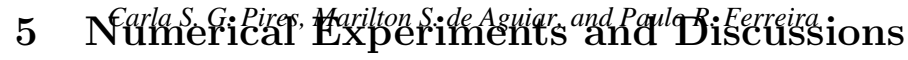

After the congestion identification method was defined, it was necessary to evaluate it in terms of precision and, consequently, the viability of the method. To that end, simulations comparing the performance of the proposed method with the algorithm $\mathrm{A}^{*}$, standard technique for the route calculation, were made. In a simulation environment developed in Netlogo, 2 (two) cars were inserted, one of them using the methodology that uses pheromones when calculating the route and the other one using pure $\mathrm{A}^{*}$ algorithm. In addition, experiments were performed for different amounts of cars of random behaviour for the evaluation in distinct congestion situations (as shown in Table 2). The parameterization was made according to [10] that also used ACO for congestion prediction, where values of 0.7 and 0.8 for evaporation and deposit respectively were pointed as good.

After 6000 simulations of the model, it was possible to observe that the average time (in ticks of simulation) of completion of route, when considering the weight of the pheromone in the cost of the Heuristic $\mathrm{A}^{*}$ is lower if compared to the route without considering the pheromone for all congestion situations.

It can be observed that the number of cars used in the simulations influence the average times obtained for calculating the best route. As the number of cars increases, the average time for the calculation also increases, thus, the longer times were obtained when 600 cars were used. The average times presented in Table 2 shows that in all simulations the time of the route using pheromones were lower in relation to the simulations not using pheromones. It could be observed in the simulations that situations where the congestion rate is high, to seek for alternative routes may lead to other congestion situations, which can be worse than waiting for the normalization of the flow. Thus, it was verified that the proposed method is effective.

Subsequent experiments for calibration of the parameters were made, such as increment and decrement of pheromones, weight of the pheromones, weight of the distance to cover all the way, evaporation of pheromone, pheromones limit. Also, experiments were performed to verify the minimum percentage of the number of cars depositing pheromones that makes the model more effective.

The methodology of the tests was systematized in order to compose the best set of parameters for the model. The systematization and planning of experiments was necessary to make possible the evaluation of the behavior of the evaluated parameters. In this sense, an analysis of variance was performed using for this, the full factorial design.

Were analyzed to each experiment 2 factors at 3 levels, so we will have a planning where all parameters are studied in three levels thus have a factorial design $2^{3}$ with a planning matrix with 9 experiments.

For planning matrices presented in Figure 2 the following response surfaces were obtained.

Considering the data of the correlation between weight of pheromone and weight of distance, was obtained on analysis of variance in Table 3 , where we can observe that the values of $p$ are smaller than 0.05 , this means that factors are considered significant, ie, both factors are relevant in the media presented.

Observing the results presented in Table 3, it is possible to identify that the best results were obtained with the parameterization Weight of the Pheromone 0.7, Weight of Distance 0.5 and Evaporation 0.3. Also, it can be observed that the highest average times were obtained when the decrements used for Evaporation were higher. This behavior can be attributed to the fact that the algorithm considers without congestion a stretch that is still crowded, and calculate a route that leads the vehicle to get congested rather than calculate a route that avoids a particular stretch. Thus, this parameterization was used in the other simulations. 
Table 2: Efficiency of the pheromone model.

\begin{tabular}{llll}
\hline & Number of cars With pheromones Without pheromones \\
\hline 200 & $\mathbf{3 6 8 . 3 7}$ & 426.91 \\
Average time (ticks) & 400 & $\mathbf{4 8 8 . 4 8}$ & 542.52 \\
600 & $\mathbf{4 9 1 . 1 4}$ & 572.81 \\
\hline
\end{tabular}

\begin{tabular}{|c|c|c|c|c|}
\hline \multicolumn{5}{|c|}{2 factors } \\
\hline \multirow{2}{*}{\multicolumn{2}{|c|}{$\begin{array}{l}\text { Weight pheromone } \\
\text { Weight distance }\end{array}$}} & 0,3 & & 0,7 \\
\hline & & 0,3 & & 0,7 \\
\hline xperiments & & WP & WD & Time \\
\hline 1 & $-1,-1$ & 0,3 & 0,3 & 445,32 \\
\hline 2 & $-1,0$ & 0,3 & 0,5 & 372,59 \\
\hline 3 & $-1,+1$ & 0,3 & 0,7 & 405,22 \\
\hline 4 & $0,-1$ & 0,5 & 0,3 & 427,59 \\
\hline 5 & $0,+1$ & 0,5 & 0,7 & 419,54 \\
\hline 6 & $+1,-1$ & 0,7 & 0,3 & 404,65 \\
\hline 7 & $+1,0$ & 0,7 & 0,5 & 334,45 \\
\hline & $+1,+1$ & 0,7 & 0,7 & 423,95 \\
\hline & 0,0 & 0,5 & 0,5 & 398,35 \\
\hline
\end{tabular}

(a)

\begin{tabular}{|l|l|l|l|}
\hline \multicolumn{1}{|c|}{ 2 factors } & -1 & 0 & +1 \\
\hline Weight pheromone & 0,3 & 0,5 & 0,7 \\
\hline Evaporation & 0,1 & 0,3 & 0,5 \\
\hline
\end{tabular}

\begin{tabular}{|l|l|l|l|l|l|}
\hline Experiments & \multicolumn{2}{|l|}{} & WP & E & Time \\
\hline 1 & -1 & -1 & 0,3 & 0,1 & 473,2 \\
\hline 2 & -1 & 0 & 0,3 & 0,3 & 421,95 \\
\hline 3 & -1 & +1 & 0,3 & 0,5 & 433,72 \\
\hline 4 & $0-$ & 1 & 0,5 & 0,1 & 425,52 \\
\hline 5 & 0 & +1 & 0,5 & 0,5 & 394,72 \\
\hline 6 & +1 & -1 & 0,7 & 0,1 & 399,91 \\
\hline 7 & +1 & 0 & 0,7 & 0,3 & 375,1 \\
\hline 8 & +1 & +1 & 0,7 & 0,5 & 404,5 \\
\hline 9 & 0 & 0 & 0,5 & 0,3 & 455,4 \\
\hline
\end{tabular}

(b)

\begin{tabular}{|l|l|l|l|}
\hline \multicolumn{1}{|c|}{ 2 factors } & -1 & 0 & +1 \\
\hline Evaporation & 0,1 & 0,3 & 0,5 \\
\hline Weight distance & 0,3 & 0,5 & 0,7 \\
\hline
\end{tabular}

\begin{tabular}{|l|l|l|l|l|}
\hline Experiments & & WD & E & Time \\
\hline 1 & $-1,-1$ & 0,3 & 0,1 & 417,55 \\
\hline 2 & $-1,0$ & 0,3 & 0,3 & 404,65 \\
\hline 3 & $-1,+1$ & 0,3 & 0,5 & 420,2 \\
\hline 4 & $0,-1$ & 0,5 & 0,1 & 366,00 \\
\hline 5 & $0,+1$ & 0,5 & 0,5 & 385,86 \\
\hline 6 & $+1,-1$ & 0,7 & 0,1 & 391,59 \\
\hline 7 & $+1,0$ & 0,7 & 0,3 & 482,27 \\
\hline 8 & $+1,+1$ & 0,7 & 0,5 & 478,85 \\
\hline 9 & 0,0 & 0,5 & 0,3 & 334,45 \\
\hline
\end{tabular}

(c)

Fig. 2: Planning matrix: (a) Weight pheromone $\mathrm{x}$ Weight distance; (b) Weight pheromone $\mathrm{x}$ Evaporation; (c) Weight distance $\mathrm{x}$ Evaporation.

\begin{tabular}{|c|c|c|c|c|c|c|c|c|c|c|}
\hline \multirow[b]{2}{*}{ Variable } & \multicolumn{10}{|c|}{\begin{tabular}{|l} 
T-test for Dependent Samples (pfpd) \\
Marked differences are significant at $p<, 05000$
\end{tabular}} \\
\hline & \begin{tabular}{|l|} 
Mean \\
\end{tabular} & Std.Dv. & $\mathrm{N}$ & Diff. & $\begin{array}{l}\text { Std.Dv. } \\
\text { Diff. }\end{array}$ & $\mathrm{t}$ & df & $p$ & $\begin{array}{l}\text { Confidence } \\
-95,000 \% \\
\end{array}$ & $\begin{array}{l}\text { Confidence } \\
+95,000 \%\end{array}$ \\
\hline PF & 0,5000 & 0,17321 & & & & & & & & \\
\hline TEMPO & 403,5178 & 33,08862 & 9 & $-403,018$ & 33,13444 & $-36,4893$ & 8 & 0,000000 & 377,5484 & 428,4872 \\
\hline PD & 0,5000 & 0,17321 & & & & & & & & \\
\hline TEMPO & 403,5178 & 33,08862 & 9 & $-403,018$ & 33,11087 & $-36,5153$ & 8 & 0,000000 & 377,5665 & 428,4690 \\
\hline TEMPO & 4403,5178 & 33,08862 & & & & & & & & \\
\hline PF & 0,5000 & 0,17321 & 9 & 403,018 & 33,13444 & 36,4893 & 8 & 0,000000 & 377,5484 & 428,4872 \\
\hline TEMPO & $\overline{403,5178}$ & 33,08862 & & & & & & & & \\
\hline PD & 0,5000 & 0,17321 & 9 & 403,018 & 33,11087 & 36,5153 & 8 & 0,000000 & 377,5665 & 428,4690 \\
\hline
\end{tabular}

Fig. 3: Student t-test values. 
Experla S.G. Gires.AAarilton.S. Ge Aguiar, and Paulo R. Ferreiration were made once the parameterization was defined. The recalculation occurs in situations where the vehicle remains motionless for a certain period. These tests aimed to identify the effectiveness of including recalculation and also calibrate the parameter called Recalculation Limit, which determines the time limit for the route recalculation. The results are displayed in Table 4.

Observing Table 4 can be verified that the time limit 1,0 Ticks to recalculate the route presented better results.

Table 3: Analysis of parameterization with 600 cars on the environment, $100 \%$ with pheromones.

\begin{tabular}{ccccc}
\hline \multicolumn{2}{c}{ Parameterization for 6000 performances } & \multicolumn{3}{c}{ Average time (Ticks) } \\
\hline Weight pheromone Weight distance Evaporation $\mathrm{W} /$ pheromone $\mathrm{W} / \mathrm{o}$ pheromone \\
\hline 0,5 & 0,5 & 0,3 & $\mathbf{4 5 5 , 4} \pm 14,41$ & $398,35 \pm 4,64$ \\
0,5 & 0,5 & 0,1 & $\mathbf{4 2 5 , 5 2} \pm 8,31$ & $418,09 \pm 4,14$ \\
0,5 & 0,5 & 0,5 & $\mathbf{3 9 4 , 7 2} \pm 4,65$ & $408,86 \pm 6,23$ \\
0,5 & 0,3 & 0,3 & $\mathbf{4 2 7 , 5 9} \pm 3,54$ & $480,36 \pm 5,45$ \\
0,5 & 0,7 & 0,3 & $\mathbf{4 1 9 , 5 4} \pm 4,67$ & $460,09 \pm 4,41$ \\
0,5 & 0,5 & 0,3 & $\mathbf{3 9 8 , 3 5} \pm 9,24$ & $455,4 \pm 7,86$ \\
0,7 & 0,5 & 0,1 & $\mathbf{3 6 6 , 0 0} \pm 2,44$ & $399,91 \pm 4,34$ \\
0,7 & 0,5 & 0,5 & $\mathbf{3 8 5 , 8 6} \pm 3,18$ & $404,05 \pm 6,23$ \\
0,7 & 0,7 & 0,1 & $\mathbf{3 9 1 , 5 9} \pm 7,45$ & $399,27 \pm 5,87$ \\
$\mathbf{0 , 7}$ & $\mathbf{0 , 5}$ & $\mathbf{0 , 3}$ & $\mathbf{3 3 4 , 4 5} \pm \mathbf{2 , 1 4}$ & $\mathbf{3 7 5 , 1 0} \pm \mathbf{2 , 8 7}$ \\
0,7 & 0,3 & 0,3 & $\mathbf{4 0 4 , 6 5} \pm 4,67$ & $440,35 \pm 6,14$ \\
0,7 & 0,3 & 0,1 & $\mathbf{4 1 7 , 5 5} \pm 2,98$ & $430,3 \pm 4,32$ \\
0,3 & 0,3 & 0,3 & $\mathbf{4 4 5 , 3 2} \pm 3,23$ & $479,18 \pm 5,90$ \\
0,3 & 0,5 & 0,3 & $\mathbf{3 7 2 , 5 9} \pm 2,05$ & $421,95 \pm 2,97$ \\
0,3 & 0,7 & 0,3 & $\mathbf{4 0 5 , 2 2} \pm 1,65$ & $459,82 \pm 4,03$ \\
0,3 & 0,5 & 0,1 & $\mathbf{4 7 3 , 2} \pm 4,26$ & $445,25 \pm 8,90$ \\
0,3 & 0,5 & 0,3 & $\mathbf{4 2 1 , 9 5} \pm 3,89$ & $372,59 \pm 7,98$ \\
0,3 & 0,5 & 0,5 & $\mathbf{4 3 3 , 7 2} \pm 6,75$ & $392,72 \pm 5,73$ \\
\hline
\end{tabular}

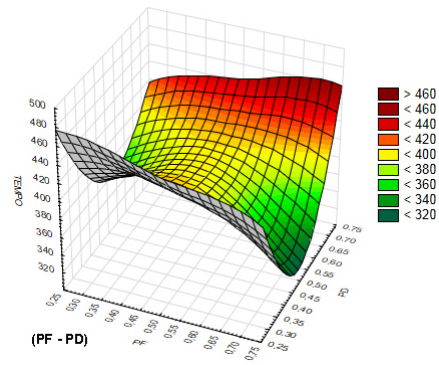

(a)

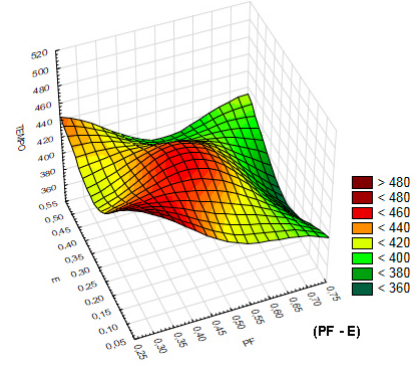

(b)

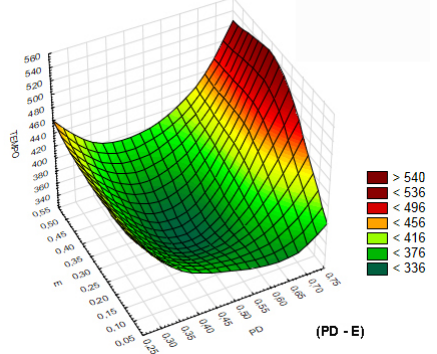

(c)

Fig. 4: Response surface graph: (a) Weight pheromone $\mathrm{x}$ Weight distance; (b) Weight pheromone $\mathrm{x}$ Evaporation; (c) Weight distance $\mathrm{x}$ Evaporation. 
Mobile ACORoute - Route Recommendation Based on Communication by Pheromones

Table 4: Analysis of decision parameters for the route recalculation.

\begin{tabular}{lll}
\hline 600 cars in the environment & \multicolumn{2}{c}{ Average time (Ticks) } \\
\hline Recalculation limit (Ticks) & With pheromones Without pheromones \\
1.5 & $\mathbf{3 6 9 . 8 7} \pm 3.52$ & $393.15 \pm 2.63$ \\
$\mathbf{1 . 0}$ & $\mathbf{3 6 2 . 1 5} \pm \mathbf{4 . 5 8}$ & $\mathbf{3 9 9 . 5 4} \pm \mathbf{2 . 4 1}$ \\
0.5 & $\mathbf{3 7 9 . 2 8} \pm 5.96$ & $381.90 \pm 2.06$ \\
\hline
\end{tabular}

\section{Conclusions and Future Work}

This work has as main contribution: the development of a methodology to predict congestion using pheromone-based communication. The results obtained in validation tests of the methodology through simulation and implementation of mobile device (ACORoute) show its viability at gaining time with its use.

The results obtained in the simulations confirm the applicability and effectiveness of the proposed method in predicting congestion, obtaining better results in time when compared to the non-use of the technique.

In general, it can be concluded that:

- The average times using pheromones are better in all cases.

- With the increase of the number of cars, the averages increase, yet the results were better using pheromones.

- With the introduction of the recalculation, the average was better.

- The application is in operation and it calculates the route avoiding locations with congestions.

- The application still needs to be optimized in some aspects.

Among the aspects raised to continue the work, we need to improve the following:

- Minimize the battery consumption;

- Testing in a real environment;

- Determine the pattern of behaviour of routes based on historical data, for situations where there is no information of pheromones;

- Implement the use of historical information in route calculation;

- Optimization of route calculation, improving the response time;

- Implement ACORoute for other platforms;

- Make download of the application available to the academic community.

\section{References}

1. Adler, J.L., Blue, V.J.: Toward the design of intelligent traveler information systems. Transportation Research Part C: Emerging Technologies 6(3), 157 - 172 (1998)

2. Ando, Y., Fukazawa, Y., Masutani, O., Iwasaki, H., Honiden, S.: Performance of pheromone model for predicting traffic congestion. In: Proceedings of the Fifth International Joint Conference on Autonomous Agents and Multiagent Systems. pp. 73-80. AAMAS '06, ACM, New York, NY, USA (2006)

3. Barbosa, H.J. (ed.): Ant colony optimization - techniques and applications. InTech Chapters published, Croatia (2013)

4. Blum, C., Merkle, D.: Swarm Intelligence: Introduction and Applications. Springer Publishing Company, Incorporated, 1 edn. (2008) $121 \quad$ Research in Computing Science 86 (2014) 


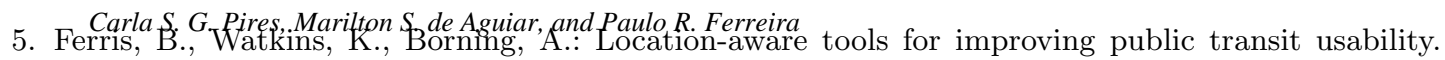
Pervasive Computing, IEEE 9(1), 13 -19 (2010)

6. Hoar, R., Penner, J., Jacob, C.: Evolutionary swarm traffic: if ant roads had traffic lights. In: Evolutionary Computation, 2002. CEC '02. Proceedings of the 2002 Congress on. vol. 2, pp. 1910-1915 (2002)

7. Kurihara, S.: Traffic-congestion forecasting algorithm based on pheromone communication model. Ant Colony Optimization - Techniques and Applications 104, 167 - 175 (2013)

8. Lucic, P., Teodorovic, D.: Transportation modeling: An artificial life approach. In: Proceedings of the 14th IEEE International Conference on Tools with Artificial Intelligence. pp. 216-. ICTAI '02, IEEE Computer Society, Washington, DC, USA (2002)

9. Lucic, P., Teodorovic, D.: Computing with bees: Attacking complex transportation engineering problems. International Journal on Artificial Intelligence Tools 12(03), 375-394 (2003)

10. Masutani, O., Ando, Y., Sasaki, H., Iwasaki, H., Fukazawa, Y., Honiden, S.: Pheromone model: Application to traffic congestion prediction. In: Brueckner, S., Marzo Serugendo, G., Hales, D., Zambonelli, F. (eds.) Engineering Self-Organising Systems. Lecture Notes in Computer Science, vol. 3910, pp. 182-196. Springer Berlin Heidelberg (2006)

11. Narzt, W., Wilflingseder, U., Pomberger, G., Kolb, D., Hrtner, H.: Self-organising congestion evasion strategies using ant-based pheromones. Iet Intelligent Transport Systems 4 (2010)

12. Ochiai, J., Kanoh, H.: Hybrid ant colony optimization for real-world delivery problems based on real time and predicted traffic in wide area road network. Fourth International conference on Computer Science and Information Technology - CCSIT 2014 4(2) (2014)

13. dos Santos, D.S., Bazzan, A.L.: Distributed clustering for group formation and task allocation in multiagent systems: A swarm intelligence approach. Applied Soft Computing 12(8), 2123 - 2131 (2012)

14. Steinmacher, I., Vieira, V., Salgado, A.C., Tedesco, P., Times, V., Ferraz, C., Huzita, E., Chaves, A.P.: The ubibus project: Using context and ubiquitous computing to build advanced public transportation systems to support bus passengers. VIII Simpsio Brasileiro de Sistemas de Informao (2012)

15. Teodorovic, D.: Swarm intelligence systems for transportation engineering: Principles and applications. Transportation Research Part C: Emerging Technologies 16(6), 651 - 667 (2008)

16. Tito, A., Borgiani, F., dos Santos, R., Tedesco, P., Salgado, A.: Contextual information in user information systems in public transportation: A systematic review. In: Intelligent Transportation Systems (ITSC), 2012 15th International IEEE Conference on. pp. 361 -366 (2012)

17. Wahle, J., Annen, O., Schuster, C., Neubert, L., Schreckenberg, M.: A dynamic route guidance system based on real traffic data. European Journal of Operational Research 131(2), $302-308$ (2001)

18. Waze: Waze mobile. https://www.waze.com/wiki/How_Waze_calculates_routes (2013) 